\title{
Cytotoxicity evaluation of symmetrically branched glycerol trimer in human hepatocellular carcinoma HepG2 cells
}

\author{
Licht Miyamoto',2, Masashi Watanabe', Mai Kono', Tsuyoshi Matsushita², \\ Hatsuhiko Hattori², Keisuke Ishizawa1, Hisao Nemoto² and Koichiro Tsuchiya1 \\ ${ }^{1}$ Department of Medical Pharmacology, Institute of Health Biosciences, The University of Tokushima Graduate \\ School, 1-78-1, Sho-machi, Tokushima 770-8505, Japan \\ 2Department of Pharmaceutical Chemistry, Institute of Health Biosciences, The University of Tokushima Graduate \\ School, 1-78-1, Sho-machi, Tokushima 770-8505, Japan
}

(Received April 24, 2012; Accepted July 21, 2012)

\begin{abstract}
An appropriate balance between lipophilicity and hydrophilicity is necessary for pharmaceuticals to achieve fine Absorption, Distribution, Metabolism and Excretion (ADME) properties including absorption and distribution, in particular. We have designed and proposed symmetrically branched oligoglycerols (BGL) as an alternative approach to improve the lipophilic-hydrophilic balance. We have previously shown that stability in circulation and water-solubility of such molecules as proteins, liposomes and hydrophobic compounds are much improved by conjugation to BGL. Albeit these successful applications of BGL, little was known whether BGL could be used in safety. Thus we conducted evaluation of the cytotoxicity of a representative BGL, symmetrically branched glycerol trimer (BGL003) in the cultured cells to clarify its biological safeness. Here we demonstrate that water-solubility of an extremely hydrophobic agent, fenofibrate was more than 2,000-fold improved just by conjugated with BGL003. BGL003 did not exhibit any significant cytotoxicity in human hepatocarcinoma HepG2 cells. Thus BGL003 should be safe and suitable strategy to endow hydrophobic molecules with much hydrophilicity.
\end{abstract}

Key words: Hydrophilicity, Lipophilicity, Cytotoxicity, Branched oligoglycerols, HepG2, Fenofibrate

\section{INTRODUCTION}

Lipophilic-hydrophilic balance is a quite important determinant of Absorption, Distribution, Metabolism and Excretion (ADME) properties of pharmaceuticals. However, it is occasionally a quite tall order to cope with both high pharmacological activities in vitro and desired solubility in drug discovery. For that reason, there have been several developed strategies to manage lipophilic-hydrophilic balance. One of the most popular and successful method is conjugating with polyethylene glycol (PEG) group by covalent bond (PEGylation) (Abuchowski and Davis, 1979). It has been successfully applied to several macromolecules such as peptides and proteins including interferons and interleukins, enhancing stability in circulation (Glue et al., 2000; Kita et al., 1990; Knauf et al., 1988). In contrast, it does not seem suitable approach for small molecules possibly because PEGs are so large molecules that it could interfere with bioactivities of the conjugated molecules. Furthermore,
PEGylation may raise cost for the synthesis partly due to its high molecular weight. Highly hydrophilic small molecules comparable to PEG would be more reasonable approach to manipulate lipophilic-hydrophilic balance. Therefore, we have designed symmetrically branched oligoglycerols (BGL) such as the trimer (BGL003) (Fig. 1A), of which molecular weight is as small as 240.25, as alternative highly hydrophilic modular molecules and have shown that stability in circulation and water-solubility of such molecules as proteins, liposomes and hydrophobic compounds were much improved by conjugation to BGL (BGLation) (Ishihara et al., 2010, 2011; Nemoto et al., 1999, 2007a, 2007b, 2010, 2011, 2012a and 2012b; Yamaguchi et al., 2008). Albeit these beneficial effects of BGLation, little was known whether BGL could be used in safety. Thus we evaluated cytotoxicity of the BGL003 using human hepatocarcinoma cells in order to evaluate biological safeness of BGL.

Correspondence: Licht Miyamoto (E-mail: licht_corresp2011@yahoo.co.jp) 
A<smiles>OCC(CO)OCC(O)COC(CO)CO</smiles>

B<smiles>CC(C)OC(=O)C(C)(C)Oc1ccc(C(=O)c2ccc(Cl)cc2)cc1</smiles>

C<smiles>CC(C)(Oc1ccc(C(=O)c2ccc(Cl)cc2)cc1)C(=O)OC(COC(CO)CO)COC(CO)CO</smiles>

Fig. 1. Structural formula of BGL003 (A), fenofibrate (B) and FF-BGL (C).

\section{MATERIALS AND METHODS}

\section{Materials and Reagents}

All reagents were analytic grade and obtained from Wako Pure Chemical (Osaka, Japan) or Kanto Chemical (Tokyo, Japan) unless otherwise stated. Fenofibrate (Fig. 1B) was purchased from Sigma-Aldrich (St. Louis, MO, USA). BGL003 (1,3-bis[(2,2-dimethyl-1,3-dioxan5-yl)oxy]-2-propanol) and fenofibrate-BGL003 conjugate (FF-BGL) (Fig. 1C) were synthesized as described elsewhere (Hattori et al., 2012; Nemoto et al., 2007b, 2010, 2012c).

\section{Cell culture and cell viability assay}

Human hepatocellular carcinoma, HepG2 cells were from American Type Culture Collection (MD, US). Cells were cultured in Dulbecco's Modified Eagle medium containing $10 \%$ fetal bovine serum in humidified atmosphere with $5 \% \mathrm{CO}_{2}$. Cell viability was assessed by MTT ((3-(4,5-di-methylthiazol-2-yl)-2,5-diphenyltetrazolium bromide) assay with modification (Mosmann, 1983). Briefly, HepG2 cells at $80 \%$ confluent in 96-well culture plate were exposed to compounds at the indicated concentration and duration in the culture medium. MTT was added at $1 \mathrm{mg} / \mathrm{ml}$ and incubated for $3 \mathrm{hr}$. Formazan formed in the cells was dissolved in $0.05 \%$ Triton X100 with $0.003 \mathrm{M}$ hydrochloric acid and Absorbance at 570/630 nm was measured using a microplate spectrophotometer, infinite M1000, (Tecan, Mannedorf, Switzerland). Cell viability was calculated according to the standard values of wells without cells as $0 \%$ and those without compounds as $100 \%$.

\section{Chromatographic analysis by UPLC}

Chromatographic analysis was performed by an ultrahigh performance liquid chromatography (UPLC) system, ACQUITY (Waters, Milford, MA, USA) with a reversedphase BEH C18 column (1.7 $\mu \mathrm{m}, 2.1 \mathrm{id}$ x $50 \mathrm{~mm}$ length). Aqueous solution of $45 \%$ acetonitrile, $0.1 \%$ trifluoroacetic acid was used as a mobile phase. Separation was carried out at a constant flow rate at $1.0 \mathrm{ml} / \mathrm{min}$ with measuring absorbance at $254 \mathrm{~nm}$.

\section{Evaluation of octanol-water partition coefficient}

The octanol-water partition coefficient value $(\mathrm{Po} / \mathrm{w})$ was determined according to the standard protocol of Japanese National Institute of Technology and Evaluation based on Organization for Economic Co-operation and Development (OECD) Guidelines for the Testing of Chemicals (OECD, 1995). Briefly, fenofibrate or FF-BGL were solved in water-saturated 1-octanol and vigorously shaken for $2 \mathrm{hr}$ in the same volume of water. Concentration of the compound in each layer separated by centrifuge was determined by UPLC.

\section{Estimation of water-solubility value}

Excessive amount of fenofibrate or FF-BGL in water was vigorously sonicated for $5 \mathrm{~min}$ at 25 degrees centigrade in ultrasonic bath. Insoluble matters were removed by centrifugation $(21,500 \mathrm{x} \mathrm{g}$, for $5 \mathrm{~min})$ and following filtration through $0.45 \mu \mathrm{m}$ pores (Millipore, Billerica, MA, USA). Concentrations of compounds in the aqueous solution were determined by UPLC.

\section{Statistical analyses}

Values were expressed as means \pm S.E. Comparisons between groups were evaluated by analysis of variance (ANOVA) and Tukey's test. $\mathrm{P}<0.01$ was considered statistically significant.

\section{RESULTS AND DISCUSSION}

We have previously shown that BGLation strikingly improved physical properties including hydrophilicity of lipophilic molecules (Ishihara et al., 2010, 2011; Nemoto et al., 2007a, 2011, 2012a, 2012b; Yamaguchi et al., 2008). Recently we succeeded in synthesizing a novel molecule, BGL003-conjugated fenofibrate (FF-BGL) (Fig. 1C) (Nemoto et al., 2012c). Fenofibrate is a popular peroxisome proliferator-activated receptor (PPAR) $\alpha$ agonist clinically used as an anti-hyperlipidemic drug and is known for its exceptionally hydrophobic character. The striking decrease in retention time in chromatographic separation by reversed phase UPLC clearly revealed 


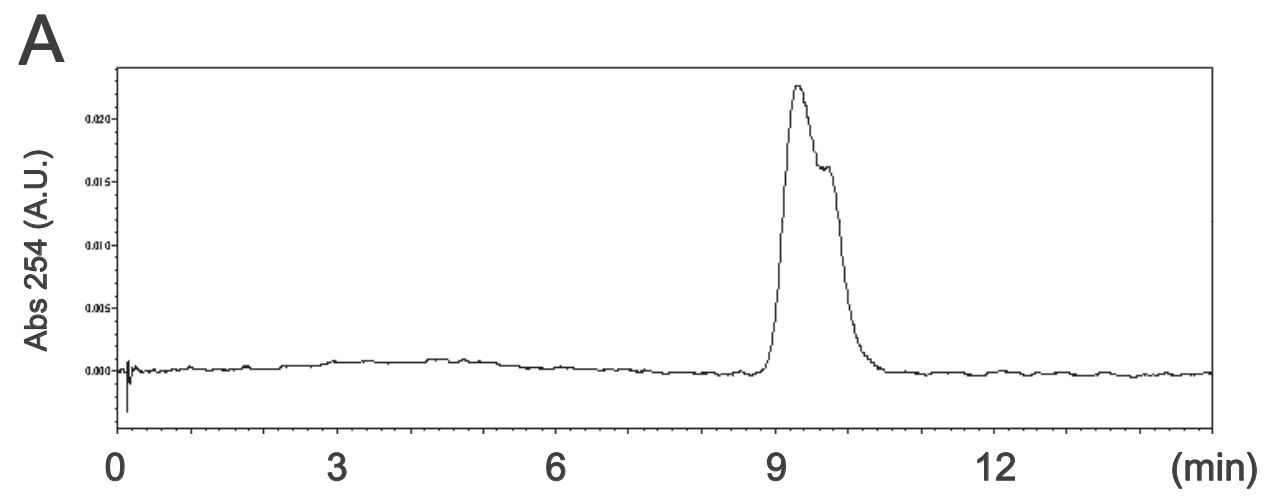

B
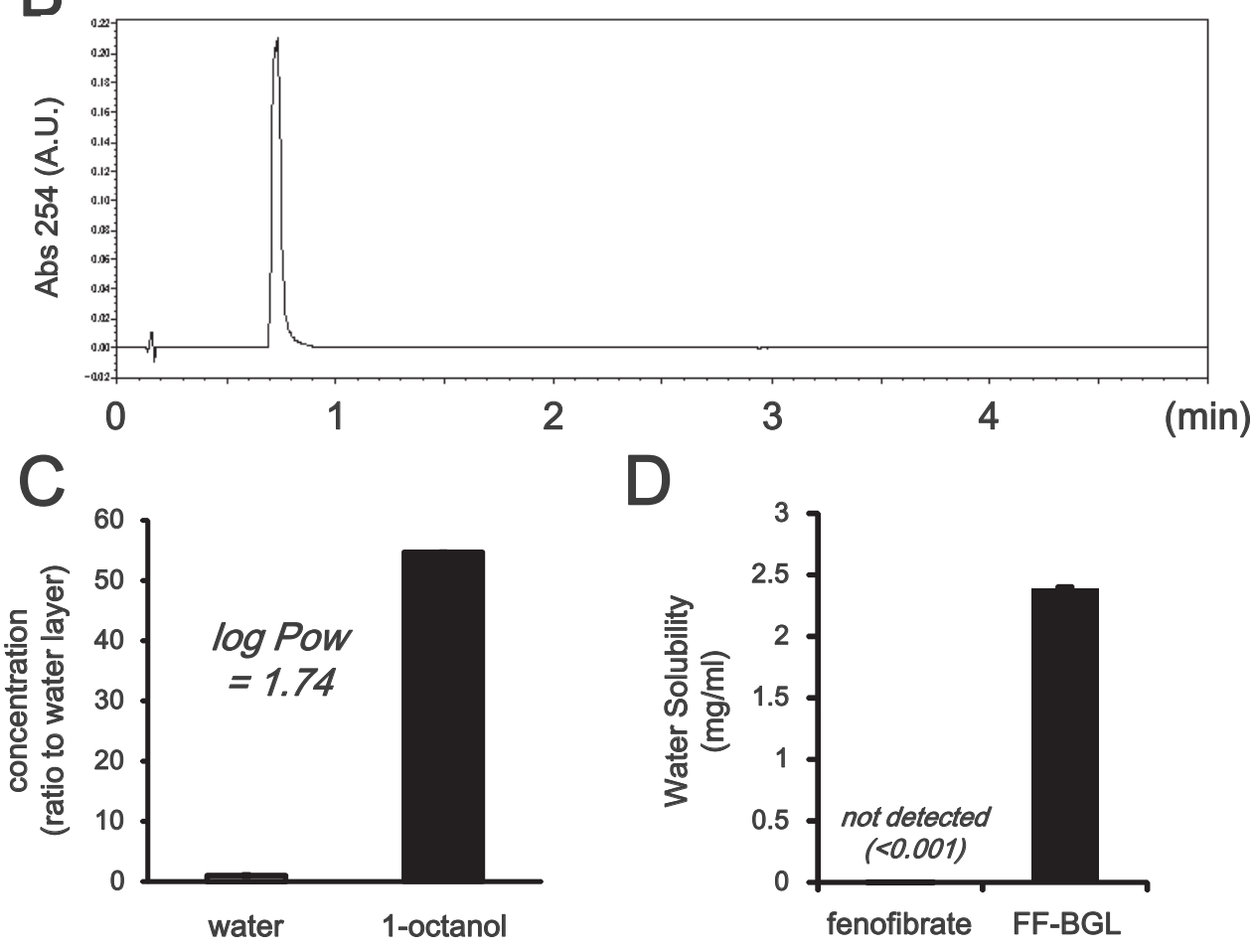

Fig. 2. Improvement of hydrophilicity by BGLation. Chromatographic separation of fenofibrate (A) and FF-BGL (B) by reversed phase UPLC. Distribution of the FF-BGL between the same amount of 1-octanol and water, and calculated octanol-water partition coefficient (C). Water-solubility of fenofibrate and FF-BGL (D). Representative data (A, B), n = $5(C, D)$.

that FF-BGL is far hydrophilic than original fenofibrate (Figs. 2A, B). We next tried to determine Po/w of these compounds, which is a standard strategy to evaluate the lipophilicity-hydrophilicity balance. However, fenofibrate is so hydrophobic that we could not detect it in the water layer by partition with 1-octanol nor determine the $\mathrm{Po} / \mathrm{w}$. In contrast, the $\log \mathrm{Po} / \mathrm{w}$ of FF-BGL was measurable and calculated as 1.74 (Fig. 2C). A previous report using atom/fragment contribution values estimated the log Po/w of fenofibrate as 5.19 (Meylan and Howard, 1995), suggesting that the hydrophilicity of fenofibrate was much improved by BGLation. In accordance, estimated water-solubility values of fenofibrate and FF-BGL were below detectable level $(<0.001 \mathrm{mg} / \mathrm{ml})$ and $2.4 \mathrm{mg} / \mathrm{ml}$, each, clearly demonstrating more than 2,000-times improvement of hydrophilicity of fenofibrate by BGLa- 
A

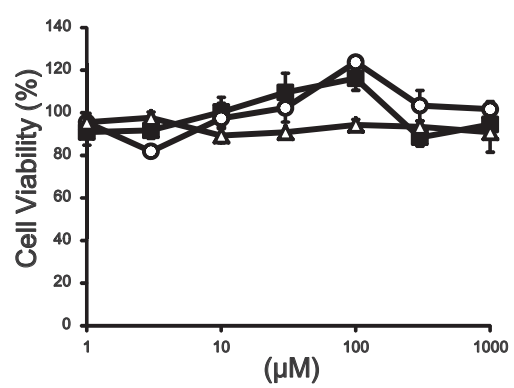

B

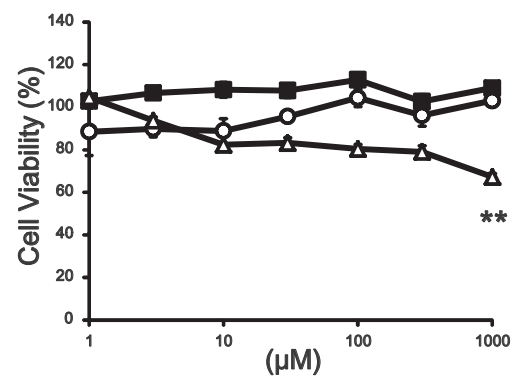

C

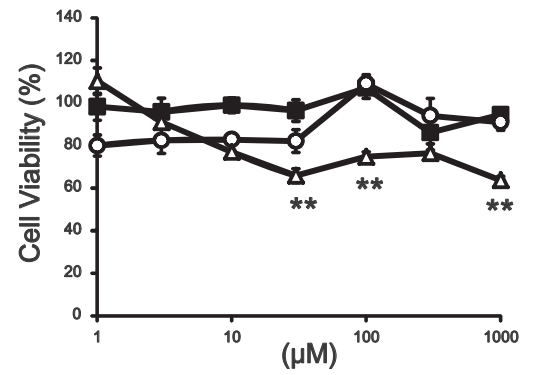

Fig. 3. Cytotoxicity of BGL003. Viability of HepG2 cells exposed to BGL003 (closed square), glycerol (open circle) or 5-FU (closed triangle) at indicated concentration for $24 \mathrm{hr}(\mathrm{A}), 48 \mathrm{hr}(\mathrm{B})$ or $72 \mathrm{hr}(\mathrm{C})$. **; ${ }^{*}<0.01$ vs no treatment (expressed as $100 \%$ cell viability), $\mathrm{n}=4$.

tion (Fig. 2D). Fenofibrate is one of the most hydrophobic agents among the pharmaceuticals currently clinically used as the estimated $\log \mathrm{Po} / \mathrm{w}$ of fenofibrate is one of the highest one (Meylan and Howard, 1995). Thus BGLation of fenofibrate may be beneficial to its ADME properties by ameliorating absorption and distribution. We also preliminarily confirmed that lipid-lowering effect of FFBGL was not impaired using normal rats (Nemoto et al., 2012c). Further study on ADME profiles of FF-BGL is required to clarify comprehensive pharmacological benefit of BGLation of fenofibrate.

Albeit the merits of BGLation on the water-solubility, little was known whether BGL003 could be used safely. Thus we determined cytotoxicity of BGL003 in order to evaluate biological safety of BGL003. We used human hepatocarcinoma HepG2 cells as we expected that this cell line could enable us to simultaneously assess the cytotoxicity of BGL003 and its possible metabolites produced in the human liver. The viability of HepG2 cells were almost unchanged when exposed to BGL003 as well as monomeric glycerol at the concentration of $1 \sim 1,000 \mu \mathrm{M}$ for 24 72 hr (Fig. 3). In contrast, 30, 100 and 1,000 $\mu \mathrm{M}$ of 5-fluorouracil (5-FU) significantly reduced the cells alive since $72 \mathrm{hr}$ after treatments, though it did not show any cytotoxicity within $24 \mathrm{hr}$ of the exposure (Fig. 3). It took a certain time for 5-FU, a typical antimetabolite drug, to exhibit its cytotoxicity assumably because 5-FU needed to be converted into nucleotide form and incorporated DNA synthesis, leading to cell cycle arrest (reviewed in (Pinedo and Peters, 1988)). It is noteworthy that BGL003 did not cause any apparent effects on the cell viability even under such high concentration around submillimolar and so long duration of exposure as the antimetabolite drug to exhibit cytotoxicity. BGL003 was as innoxious as glycerol which is widely used in miscellaneous commodities around us including foods and medicines. Our study on acute oral toxicity of BGL003 evaluated in normal mice will be reported in the accompanying paper (Miyamoto et al., in press).

There is no way to determine whether BGL is taken up into the cells at the moment. BGL003 is quite difficult to detect because it has no characteristic chemical functional groups but hydroxyl groups nor specific ultraviolet absorption. BGL is thought to be too hydrophilic to easily penetrate plasma membrane. Thus we do not suppose that BGL003 should enter into the cells at the moment. Further study using an isotopic label will provide us much information including all aspects of ADME properties.

In conclusion, we demonstrated that conjugation with BGL003 strikingly ameliorated hydrophilicity of such quite hydrophobic compound as fenofibrate, and that BGL003 did not exhibit apparent toxic effect against the cultured hepatocarcinoma cells. BGL should be safe and suitable alternative to endow hydrophobic molecules with much hydrophilicity.

\section{ACKNOWLEDGMENTS}

We thank Hiroko Hiraoka and Kazuyo Fukumoto for secretarial assistance, Chiaki Taoka for contribution to the preliminary experiments, Valentino Milton for checking the manuscript and Drs. Toshiaki Tamaki and Kazuhiko Tsutsumi for helpful advice. This work was partly supported by the Japan Science and Technology Agency (JST), Adaptable and Seamless Technology Transfer Program through Target-driven R\&D in 2009-2012. 
Hepatotoxicity evaluation of branched glycerol trimer

\section{REFERENCES}

Abuchowski, A. and Davis, F.F. (1979): Preparation and properties of polyethylene glycol-trypsin adducts. Biochim. Biophys. Acta., 578, 41-46.

Glue, P., Fang, J.W., Rouzier-Panis, R., Raffanel, C., Sabo, R., Gupta, S.K., Salfi, M. and Jacobs, S. (2000): Pegylated interferon-alpha2b: pharmacokinetics, pharmacodynamics, safety, and preliminary efficacy data. Hepatitis $\mathrm{C}$ Intervention Therapy Group. Clin. Pharmacol. Ther., 68, 556-567.

Hattori, H., Matsushita, T., Yoshitomi, K., Katagiri, A. and Nemoto, H. (2012): An efficient method for the refinement of 1,3-methyleneglycerol via bridged acetal exchange and synthesis of the symmetrically branched glycerol trimer. Synthesis, 44, 23652373.

Ishihara, A., Yamauchi, M., Kusano, H., Mimura, Y., Nakakura, M., Kamiya, M., Katagiri, A., Kawano, M., Nemoto, H., Suzawa, T. and Yamasaki, M. (2010): Preparation and properties of branched oligoglycerol modifiers for stabilization of liposomes. Int. J. Pharm., 391, 237-243.

Ishihara, A., Yamauchi, M., Tsuchiya, T., Mimura, Y., Tomoda, Y., Katagiri, A., Kamiya, M., Nemoto, H., Suzawa, T. and Yamasaki, M. (2011): A novel liposome surface modification agent that prolongs blood circulation and retains surface ligand reactivity. J. Biomater. Sci. Polym. Ed., in press.

Kita, Y., Rohde, M.F., Arakawa, T., Fagin, K.D., Fish, E.N. and Banerjee, K. (1990): Characterization of a polyethylene glycol conjugate of recombinant human interferon-gamma. Drug Des. Deliv., 6, 157-167.

Knauf, M.J., Bell, D.P., Hirtzer, P., Luo, Z.P., Young, J.D. and Katre, N.V. (1988): Relationship of effective molecular size to systemic clearance in rats of recombinant interleukin-2 chemically modified with water-soluble polymers. J. Biol. Chem., 263, 1506415070.

Meylan, W.M. and Howard, P.H. (1995): Atom/fragment contribution method for estimating octanol-water partition coefficients. J. Pharm. Sci., 84, 83-92.

Miyamoto, L., Watanabe, M., Tomida, Y., Kono, M., Fujii, S., Matsushita, T., Hattori, H., Ishizawa, K. and Nemoto, H. (2012): Acute oral toxicity evaluation of symmetrically branched glycerol trimer in ddY mice. J. Toxicol. Sci., in press.

Mosmann, T. (1983): Rapid colorimetric assay for cellular growth and survival: application to proliferation and cytotoxicity assays. J. Immunol. Methods, 65, 55-63.

Nemoto, H., Kikuishi, J., Yanagida, S., Kawano, T., Yamada, M., Harashima, H., Kiwada, H. and Shibuya, M. (1999): Design and synthesis of cholestane derivatives bearing a cascade-type poly$\mathrm{ol}$ and the effect of their property on a complement system in rat serum. Bioorg. Med. Chem. Lett., 9, 205-208.

Nemoto, H., Araki, T., Kamiya, M., Kawamura, T. and Hino, T. (2007a): A quantitative investigation of the water-solubilizing properties of branched oligoglycerols. Eur. J. Org. Chem., 18, 3003-3011.

Nemoto, H., Kamiya, M., Minami, Y., Araki, T. and Kawamura, T. (2007b): Synthesis of branched heptaglycerol bearing eight hydroxyl groups with four cyclic protecting groups. Synlett, 2091-2095.

Nemoto, H., Kamiya, M., Nakamoto, A., Katagiri, A., Yoshitomi, K., Kawamura, T. and Hattori, H. (2010): An efficient and practical method for the preparation of a branched oligoglycerol with acetonide protection groups. Chem. Lett., 39, 856-857.
Nemoto, H., Ishihara, A., Araki, T., Katagiri, A., Kamiya, M., Matsushita, T., Hattori, H., Mimura, Y., Tomoda, Y. and Yamasaki, M. (2011): Improved performance by replacing iminodiacetic residues with glyceryl residues in symmetrically branched oligoglycerols. Bioorg. Med. Chem. Lett., 21, 47244727.

Nemoto, H., Katagiri, A., Kamiya, M., Kawamura, T., Matsushita, T., Matsumura, K., Itou, T., Hattori, H., Tamaki, M., Ishizawa, K., Miyamoto, L., Abe, S. and Tsuchiya, K. (2012a): Synthesis of Paclitaxel-BGL Conjugates. Bioorg. Med. Chem., 20, 55595567.

Nemoto, H., Katagiri, A., Kamiya, M., Matsushita, T., Hattori, H., Matsumura, K., Itou, T., Kawamura, T., Kita, T., Nishida, H. and Arakaki, N. (2012b): Synthesis and Evaluation of Water-soluble Resveratrol and Piceatannol via BGLation. Bioorg. Med. Chem. Lett., 22, 5051-5054.

Nemoto, H., Kamiya, M., Nakamoto, A., Matsushita, T., Matsumura, K., Hattori, H., Kawamura, T., Taoka, C., Abe, S., Ishizawa, K., Miyamoto, L. and Tsuchiya, K. (2012c): Synthesis of Highly Water-soluble Fibrate Derivatives via BGLation. Bioorg. Med. Chem. Lett., 22, in press.

OECD (1995): Test No. 107: Partition Coefficient (n-octanol/water): Shake Flask Method. In OECD Guidelines for the Testing of Chemicals, Section 1. 107, pp.1-4. OECD Publishing, Paris.

Pinedo, H.M. and Peters, G.F. (1988): Fluorouracil: biochemistry and pharmacology. J. Clin. Oncol., 6, 1653-1664.

Yamaguchi, H., Suzawa, T., Araki, T., Kamiya, M., Nemoto, H. and Yamasaki, M. (2008): Modification of protein with BGL06, a novel branched oligoglycerol derivative. Biochim. Biophys. Acta., 1780, 680-686. 